\title{
Kinetics and mechanisms of crystal growth and diffusion in a glass-forming liquid
}

\author{
Marcio Luis Ferreira Nascimento, ${ }^{\text {a) }}$ Eduardo Bellini Ferreira, and Edgar Dutra Zanotto \\ Vitreous Materials Laboratory, Department of Materials Engineering, Federal University of São Carlos, \\ 13.565-905, São Carlos-São Paulo, Brazil
}

(Received 24 May 2004; accepted 12 August 2004)

\begin{abstract}
Extensive data on the viscosity, covering 15 orders of magnitude, and crystal growth rate, covering seven orders of magnitude, of liquid diopside $\left(\mathrm{CaO} \cdot \mathrm{MgO} \cdot 2 \mathrm{SiO}_{2}\right)$ were collected in a wide range of undercoolings from $1.10 T_{g}$ to $0.99 T_{m}\left(T_{g}\right.$ is the glass transition temperature and $T_{m}$ the melting point). The raw growth rate data were corrected for the increased interfacial temperature produced by the heat released during crystallization. A detailed analysis confirms that growth mediated by screw dislocations reasonably explain the experimental data in these wide ranges of temperatures and growth rates. Effective diffusion coefficients were calculated from crystal growth rates and from viscosity, and were then compared with measured self-diffusion coefficients of silicon and oxygen in diopside melt. The results show that oxygen and silicon control the diffusion dynamics involved in crystal growth and viscous flow. This study not only unveils the transport mechanism in this complex liquid, but also validates the use of viscosity (through the Stokes-Einstein or the Eyring equations) to account for the kinetic term of the crystal growth expression in a wide range of temperatures. (C) 2004 American Institute of Physics. [DOI: 10.1063/1.1803813]
\end{abstract}

\section{INTRODUCTION}

A recent study ${ }^{1,2}$ demonstrates that below the glass transition temperature $T_{g}$ atomic motion through a metallic glass involves single-atom hopping, whereas motion above $T_{g}$ is more collective. Other authors ${ }^{3}$ previously observed such substantial change in diffusion mechanism for organic and Lennard-Jones liquids, but occurring at higher temperatures, i.e., at about $1.2 T_{g}-1.3 T_{g}$, and this temperature has been denominated decoupling temperature $T_{d}$. Similar change in diffusion mechanism at $T_{d}>T_{g}$ is also suggested to occur for undercooled oxide liquids, but has not been firmly proved yet. In addition, a key question is, which ions or "molecular units" move collectively above $T_{g}$ or $T_{d}$ and control important kinetic phenomena, such as viscous flow and crystallization?

In this paper, we propose and demonstrate a route to infer which ions move jointly and control the crystal growth kinetics in undercooled liquids by analyzing their effective diffusion coefficient in three distinct ways for diopside $\left(\mathrm{CaO} \cdot \mathrm{MgO} \cdot 2 \mathrm{SiO}_{2}\right)$ liquid. This is an important mineral for geological studies and is also a good glass former. Several authors have determined diopside crystal growth rates for different undercoolings. ${ }^{4-8}$ However, none of these previous studies analyzed the crystal growth kinetics in a wide range of temperatures. Here we collect and combine viscosity and crystal growth data, including our own, over a very wide temperature range (from $1.10 T_{g}$ to $0.99 T_{m}$ ). In addition, the corresponding thermodynamic and kinetic data, such as the Gibbs free energy of crystallization $\Delta G$ and viscosity $\eta$ are available for diopside, which substantially help with the

\footnotetext{
a) Author to whom correspondence should be addressed. Electronic mail: pmlfn@iris.ufscar.br
}

analysis. An important motivating factor is that (hard to measure) self-diffusion coefficients of oxygen $D_{\mathrm{O}}$ and particularly silicon $D_{\mathrm{Si}}$-the slowest diffusing species in silicatesare available for liquid diopside. We then compare these actual diffusion data with effective diffusion coefficients calculated from the growth kinetics $D_{u}$ and independently from viscosity $D_{\eta}$ (using the Stokes-Einstein and the Eyring equations). To the best of our knowledge, this is the first time the diffusion mechanism that controls crystal growth kinetics in glass-forming liquids is analyzed in detail, over such a wide range of undercoolings, using independent, reliable experimental data on the thermodynamic driving force, viscosity, diffusion coefficients, and crystal growth rates.

\section{ANALYSIS OF CRYSTAL GROWTH KINETICS}

Three phenomenological models are normally employed to describe interface controlled crystal growth processes in inorganic glasses: normal growth, screw dislocation growth, and two-dimensional (2D) surface nucleated growth. According to Jackson's treatment of the interface, materials with high melting entropy ( $>4 R$, where $R$ is the gas constant), such as diopside $\left(\Delta S_{m} \equiv 10 R\right)$, are expected to exhibit crystal growth kinetics of the form predicted either by the 2D surface nucleated growth or by the screw dislocation model..$^{9,10}$

Crystallization is an exothermic process and knowledge of the crystal/liquid interface temperature is essential for analyzing crystal growth kinetics. Based on direct measurements for several glasses, Herron and Bergeron ${ }^{11}$ suggested and successfully tested empirical equations to estimate the liquidcrystal interface temperature for temperatures near the maximum crystal growth rate $u_{\max }$ and relatively far from it ( $u$ $\left.<0.67 u_{\max }\right)$. For diopside, these temperature corrections 
TABLE I. Physical parameters and equations used in this paper.

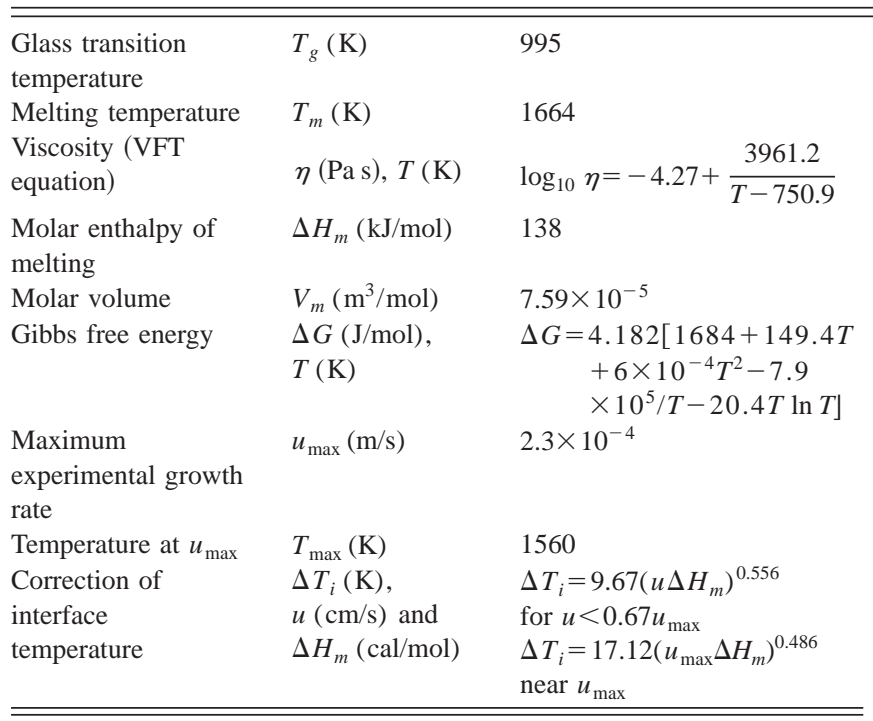

reach about $50{ }^{\circ} \mathrm{C}$ in the range of maximum growth rate, and may in principle exert a significant effect on the kinetics. Such corrections of interface temperature (see the equations in Table I) are used throughout the paper, but calculations performed with and without them demonstrate that the main conclusions would not change.

In principle, the high melting entropy of diopside $(>4 R)$ excludes the normal growth model. However, to be on the safe side, we first tested all the growth models. In the normal growth model, the surface is considered atomically rough and the degree of roughness is independent on the temperature. We found that to fit the growth rate data assuming this particular mechanism, the diameter of the diffusing building molecules $\lambda$ is $15 \AA$ if one assumes diffusivity given by the Stokes-Einstein expression, or $47 \AA$ if one assumes the Eyring expression (please refer to the Eqs. (4a) and (4b), and explanations therein). These values of $\lambda$, however, are about one order of magnitude higher than expected. We then tested the 2D surface nucleated growth model, where the surface is considered atomically smooth and defect free. In this model, growth occurs by the formation of twodimensional nuclei on top of primary crystals, which grow laterally. ${ }^{9,10}$ The 2D growth model was also discarded because a plot of $\ln (u \eta)$ versus $(T \Delta G)^{-1}$ should give a straight line, but such test has shown that a single straight line cannot fit the data. ${ }^{12}$ Kirkpatrick, Robinson, and Hays ${ }^{7}$ also discarded this model in their analysis of crystal growth kinetics at low undercoolings.

Let us, therefore, focus on the remaining model. According to the screw dislocation growth model, the crystal-liquid interface is smooth, albeit imperfect on atomic scale, and growth takes place at step sites provided by screw dislocations. The corresponding temperature-dependent growth rate $u$ may be expressed by ${ }^{9,10}$

$$
u=f \frac{D_{u}}{\lambda}\left[1-\exp \left(-\frac{|\Delta G|}{R T}\right)\right],
$$

where $D_{u}$ is an effective diffusion coefficient $\left(\mathrm{m}^{2} / \mathrm{s}\right)$ of the (unknown) molecular species that control atomic or molecular attachment at the liquid/crystal interface; $\lambda$ is the (unknown) diameter of the diffusing building molecules $\mathrm{m}$, which is equivalent to the jump distance, the crystal lattice parameter or the unit distance advanced by the interfaceparameters usually taken in such kinetic analyses; $\Delta G$ is the free energy change upon crystallization $(\mathrm{J} / \mathrm{mol}) ; R$ is the gas constant $(\mathrm{J} / \mathrm{mol} \mathrm{K}) ; T$ is the absolute temperature $(\mathrm{K})$; and $f$ is the fraction of preferred growth sites at the interface. In general, one uses $\Delta G$ calculated by the Turnbull or Hoffman approximations. ${ }^{9,10}$ Here we were fortunate enough to have experimental data for $\Delta G$ (Ref. 13) (Table I), which lie between the values calculated by the Turnbull and Hoffman expressions. The fraction of growth sites $f$ is given by

$$
f=\frac{\lambda \Delta G}{4 \pi \sigma V_{m}}
$$

where $\sigma$ is the crystal-liquid surface energy $\left(\mathrm{J} / \mathrm{m}^{2}\right)$ and $V_{m}$ is the molar volume of the crystal $\left(\mathrm{m}^{3} / \mathrm{mol}\right)$. For normal growth $(\Delta S<2 R)$, Eq. (1) applies with $f=1$.

The crystal-liquid surface energy $\sigma$ is expressed by

$$
\sigma=\frac{\alpha \Delta H_{m} \lambda}{V_{m}},
$$

where $\alpha$ is the reduced surface energy and $\Delta H_{m}$ is the melting enthalpy $(\mathrm{J} / \mathrm{mol})$.

The diffusivity can be estimated via the Stokes-Einstein or the Eyring expressions, Eqs. (4a) and (4b), respectively (that only differ by a factor of $3 \pi$ ), assuming that the molecular motion required for interfacial rearrangements controlling crystal growth is similar to that controlling viscous flow in the bulk liquid $D_{\eta}$. In this article, we present results using both equations to calculate diffusion coefficients from viscosity data.

$$
\begin{aligned}
& D_{\eta}=\frac{k_{B} T}{3 \pi \lambda \eta} \quad(\text { Stokes-Einstein }), \\
& D_{\eta}=\frac{k_{B} T}{\lambda \eta} \quad \text { (Eyring), }
\end{aligned}
$$

where $k_{B}$ is the Boltzmann constant, $\eta$ is the shear viscosity, and $\lambda$ is the (unknown) diameter of the (unknown) diffusing molecules, which has the order of a few angstroms. It has been a matter of discussion if the Stokes-Einstein and the Eyring equations can be used for calculations of crystal growth kinetics, especially at deep undercoolings, below $T_{d}$ $\sim 1.2 T_{g}$, where it has been suggested that these equations fail, ${ }^{3,14}$ but they are considered to be valid for temperatures greater than $T_{d}$ that includes most of the range of interest in this paper.

From the above discussion, to test the governing mechanism of crystal growth, one must know the glass viscosity $\eta(T)$, the free energy change due to crystallization $\Delta G(T)$, and the temperature dependent crystal growth rate $u(T)$. 


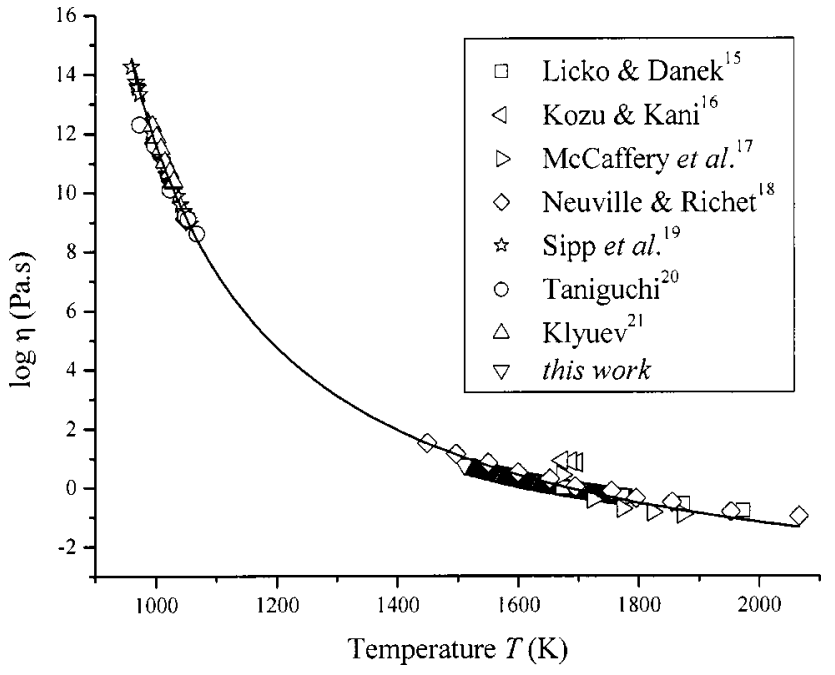

FIG. 1. Experimental viscosities of diopside glasses obtained by several authors (Refs. 15-21) and fitted VFT curve (Table I), shown as a continuous line $\left(R^{2}=0.998\right)$. Kozu and Kani's (Ref. 16) data, which clearly disagree from all the others, were not used in the fit.

\section{RESULTS AND DISCUSSION}

Figure 1 shows a collection of viscosity data from different authors ${ }^{15-21}$ including our own. A Vogel-FulcherTammann fit adheres to almost all the data in the wide temperature range considered (the resulting equation is listed in Table I). It is quite surprising that data from so many independent authors, using diopside glasses having different departures from stoichiometry and impurity levels, agree so well. We will discuss this interesting finding below.

Figure 2 shows the crystal growth rate obtained in this work and data of several authors. ${ }^{4-8}$ These combined data span a range of about seven orders of magnitude in a wide undercooling range from $1.10 T_{g}$ to $0.99 T_{m}$. Figure 2 also shows the corrected data using Herron and Bergeron's ${ }^{11}$ equations due to the increase of the temperature at the interface during crystal growth.

Analogous to the viscosity behavior, despite the disparities in glass preparation procedures, departures from stoichi-

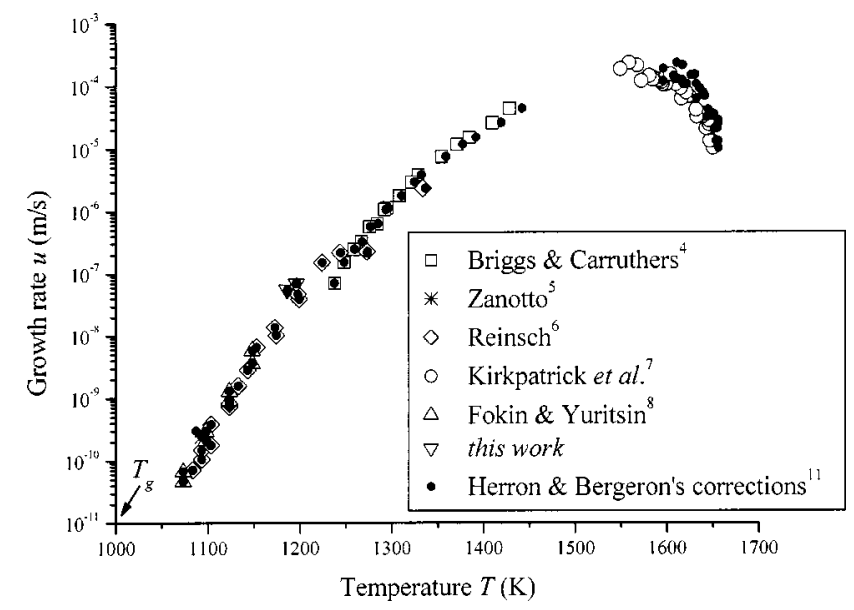

FIG. 2. Temperature dependence of the diopside growth rate in its stoichiometric melt and the same data after interface temperature corrections using the Herron and Bergeron (Ref. 11) equations.

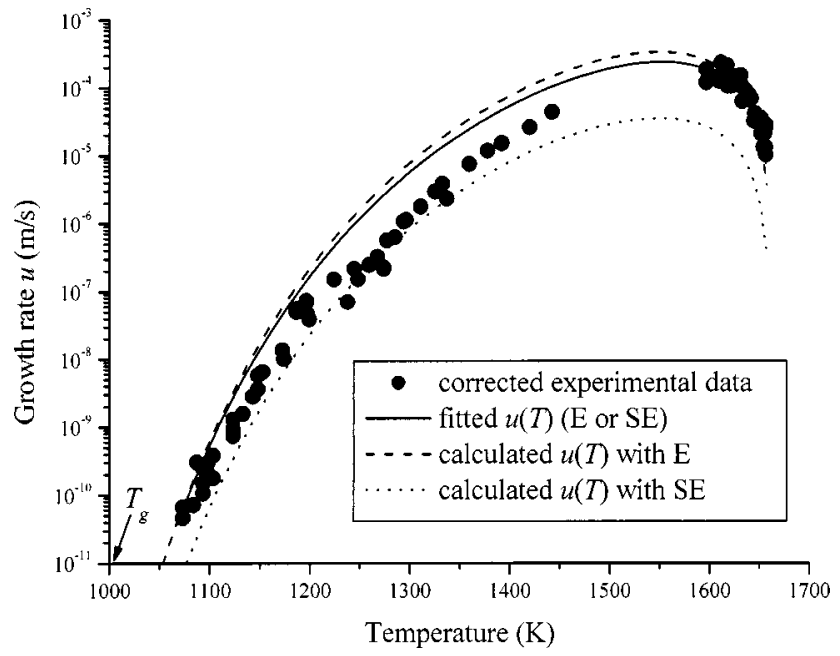

FIG. 3. Corrected experimental crystal growth rates (dots) and fitted curves (solid line) using the screw dislocation growth model and $D_{\eta}$ calculated by the Stokes-Einstein (SE) and Eyring (E) equations. Both fits coincide with $R^{2}=0.83$. The free parameter $\lambda$ is $1.04 \AA$ for $D_{\eta}$ from the Stokes-Einstein equation; and $\lambda=3.2 \AA$ for $D_{\eta}$ from the Eyring expression. Dashed and dotted lines show the calculated $u(T)$ without any adjustable parameter, considering $\lambda=2.7 \AA$, the $\mathrm{O}^{2-}$ diameter for the Stokes-Einstein and Eyring cases. Experimental data from Refs. 4-8 plus our own.

ometry, impurity levels, and methods employed for crystal growth measurements by the various investigators, the collection of experimental crystal growth rates agrees surprisingly well with respect to both their magnitude and temperature dependence indicating that for this particular composition $u$ is not very sensitive to small departures in chemical composition. Such insensitivity is probably due to the highly "depolymerized" structure of this metasilicate liquid owing to the high concentration of modifier cations $\mathrm{Ca}^{2+}$ and $\mathrm{Mg}^{2+}$. So further depolymerization does not significantly affect these kinetic properties.

For stoichiometric (polymorphic) crystallization, as in the present case, short-range molecular diffusion through the crystal/melt interface is expected to govern crystal growth. However, in most theoretical analyses of crystal growth kinetics in undercooled liquids, it is assumed that molecular transport through the interface is determined by an effective diffusion coefficient in the liquid, which is related with viscosity by the Stokes-Einstein or Eyring equations [Eqs. (4a) and (4b)]. Thus, these equations correlate the viscosity and diffusivity of the (unknown) rate-determining flow units. To analyze growth rate data one can insert Eq. (4) in Eq. (1), assuming $D_{u}=D_{\eta}$, and use the independent experimental values of $\eta(T)$ and $\Delta G(T)$, given in Table I.

However, the true size (and nature) of the diffusing "building molecules" $\lambda$ remains unknown. One can thus leave $\lambda$ as an adjustable parameter and fit Eq. (1) to the growth rate data. The fitted growth rate curves using the corrected experimental data, considering $D_{\eta}$ from the Stokes-Einstein and Eyring equations, are shown in Fig. 3. Both fits coincide with the solid line shown in Fig. 3, resulting in $\lambda=1.04 \AA$ when using the Stokes-Einstein equation [Eq. (4a)]; and $\lambda=3.20 \AA$ if the Eyring equation [Eq. (4b)] is alternatively used. These fitted values of $\lambda$ have the expected 
magnitude and are not far from the diameter of $\mathrm{Si}^{4+}(0.8 \AA)$ or $\mathrm{O}^{2-}(2.7 \AA)$, respectively. Figure 3 also shows the curves calculated with $D_{\eta}$ from the Stokes-Einstein and Eyring equations with no adjustable parameter considering, for instance, the $\mathrm{O}^{2-}$ diameter. In this case, using $D_{\eta}$ from the Eyring equation seems to give better results. Alternatively, if one uses the diameter of $\mathrm{Si}^{4+}(0.8 \AA)$, using the $D_{\eta}$ from the Stokes-Einstein equation yields better results (the curve is not shown in the Fig. 3). One can thus conclude that the fits with either Stokes-Einstein or Eyring expressions give the correct order of magnitude for $\lambda$, corroborating the validity of the present analysis. But, it should be emphasized that the fitted values of $\lambda$ carry all the errors related to the uncertainty in the other parameters of the model.

Let us then analyze the diffusion coefficients in more detail. Considering growth mediated by screw dislocations, one can isolate an effective diffusion coefficient $D_{u}$ from Eq. (1), as shown by Eq. (5), which can be estimated using the experimental growth rate data and $\lambda$ from the previous fits. Our aim is to compare the effective diffusion coefficients calculated from crystal growth kinetics with those calculated by the Stokes-Einstein and Eyring relations [Eqs. (4a) and $4(\mathrm{~b})]$,

$$
D_{u}=\frac{4 \pi \alpha \lambda u \Delta H_{m}}{\Delta G}\left[1-\exp \left(-\frac{|\Delta G|}{R T}\right)\right]^{-1} .
$$

Figures 4(a) and 4(b) show a reasonably good agreement between the diffusion coefficients, $D_{u}$ calculated from crystal growth kinetics and $D_{\eta}$ calculated from viscosity by the Stokes-Einstein and Eyring equations. Most values agree within half an order of magnitude, and, most important, the calculated curves correctly describe the temperature dependence of the effective diffusion coefficients. This congruence indicates that, whatever the bond breaking and molecular reorientation mechanism required for crystallization is, it is the same as the atomic transport mechanism that controls viscous flow.

Experimental data for the diffusivities of silicon $\mathrm{S}^{4+}$ and oxygen $\mathrm{O}^{2-}$ in liquid diopside ${ }^{22-25}$ are also shown in Figs. 4(a) and 4(b) for temperatures between $1600{ }^{\circ} \mathrm{C}$ and $2000^{\circ} \mathrm{C}$ and pressures from 1 atm to $3 \mathrm{GPa}$ (no significant effect was found in Ref. 22 on the diffusion kinetics in that pressure range; and the diffusivities of $\mathrm{Si}^{4+}$ and $\mathrm{O}^{2-}$ are almost coincident). These experimental values of $D_{\mathrm{Si}}$ and $D_{\mathrm{O}}$ agree quite well with the calculated $D_{u}$ and $D_{\eta}$, but, using the best fitted values of $\lambda$ from the growth rate curves, the StokesEinstein equation gives a better quantitative description than the Eyring expression. Obviously, one can fit other values of $\lambda$ so both Eyring and Stokes-Einstein expressions give identical curves.

Dunn $^{23}$ and Shimizu and Kushiro ${ }^{24,25}$ have determined oxygen diffusion coefficients in liquid diopside and compared them with estimates using the Eyring equation. According to Dunn, ${ }^{23}$ the oxygen self-diffusion coefficients do not match those estimated by the Eyring equation unless $\lambda$ was larger than the diameter of oxygen anion (taken as 2.6 $\AA$ ). To explain this alleged failure of the Eyring equation he proposed that larger structural units, such as $\mathrm{SiO}_{4}^{4-}$ tetrahedra control viscous flow. On the other hand, Shimizu and
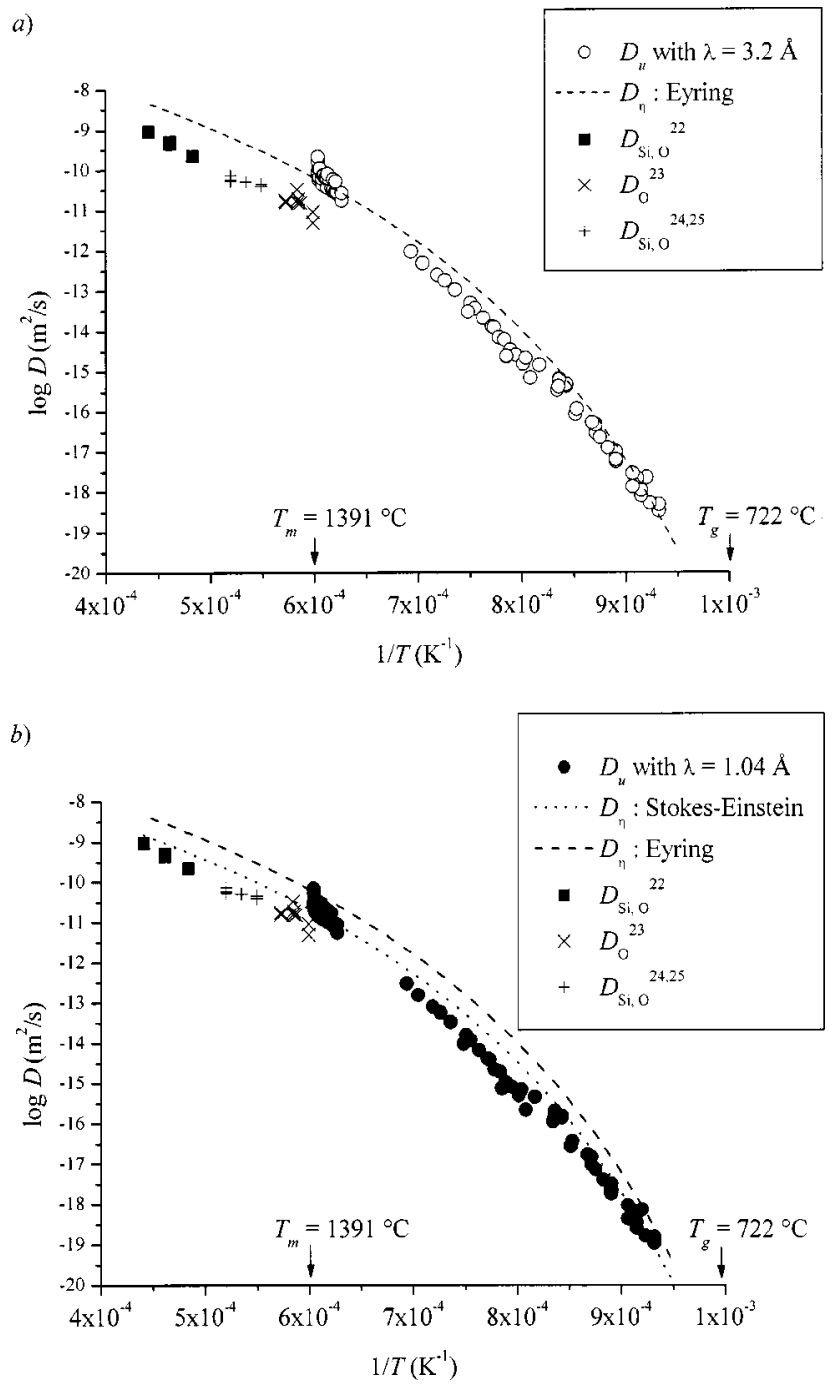

FIG. 4. Logarithm of the effective diffusion coefficient $D_{u}$ obtained from the crystal growth kinetics $[\mathrm{Eq}$. (5)] in a wide range of temperatures $\left(1.10 T_{g}<T<0.99 T_{m}\right)$ and diffusion coefficients $D_{\eta}$ calculated by the Stokes-Einstein and Eyring relations [Eqs. (4a) and (4b)], using the experimental $\eta$ and $\lambda$ from the fits to the experimental $u(T)$. (a) $D_{\eta}$ calculated by the Eyring equation with $\lambda=3.2 \AA$ ( $\lambda$ from the best fit to the crystal growth curve). The measured (Refs. 22-25) self-diffusion coefficients of $\mathrm{Si}^{4+}$ and $\mathrm{O}^{2-}$ are also shown. (b) $D_{\eta}$ calculated by the Stokes-Einstein equation with $\lambda=1.04 \AA$ ( $\lambda$ from the best fit to the crystal growth curve). The $D_{\eta}$ from Eyring is repeated for comparison, and the measured (Refs. 22-25) selfdiffusion coefficients of $\mathrm{Si}^{4+}$ and $\mathrm{O}^{2-}$ are also shown.

Kushiro $^{24}$ noted that diffusivity correlates well with viscosity, and suggested that individual oxygen ions (having diameter $\approx 2.8 \AA$ ) are the migrating species. Regarding this matter, we consider that, if one takes into account the experimental errors and the several assumptions involved in such equations, they are not sufficiently accurate to allow a precise calculation of the diameter of the diffusing molecular species. Therefore, the calculated diffusion coefficients have the same order of magnitude and reasonably agree with those of silicon and oxygen. It is also important to note that the temperature dependencies of these three independently determined diffusion coefficients match quite well. Thus, at high temperatures, the effective diffusion mechanism controlling 
crystal growth and viscous flow is related with the selfdiffusion of $\mathrm{Si}^{4+}$ and $\mathrm{O}^{2-}$.

To the best of our knowledge, there are no diffusion data of $\mathrm{Ca}^{2+}$ and $\mathrm{Mg}^{2+}$ in diopside glass or liquid but, by analogy with other silicate glasses and liquids, they probably diffuse much faster than $\mathrm{Si}^{4+}$ and $\mathrm{O}^{2-}$. Schmelzer et al. ${ }^{3}$ proposed to calculate an effective diffusion coefficient $D_{\text {eff }}$ for multicomponent glass-forming melts through Eq. (6),

$$
D_{\mathrm{eff}}=\frac{1}{\sum_{i} \frac{v_{i}^{2}}{x_{i} D_{i}}},
$$

where $D_{i}$ is the partial diffusion coefficient of the different components in the system, $x_{i}$ is the molar fraction of each component in the melt, and the parameters $v_{i}$ are the coefficients describing the stoichiometric composition of the evolving crystalline phase. In our case $x_{i}=v_{i}$. Considering $D_{\mathrm{Si}} \approx D_{\mathrm{O}}$ and $\left(D_{\mathrm{Ca}}, D_{\mathrm{Mg}}\right) \gg\left(D_{\mathrm{Si}}, D_{\mathrm{O}}\right)$, it was possible to calculate an effective coefficient $D_{\text {eff }}$, which is quite close to those of silicon and oxygen self-diffusion coefficients at 1 atm.

We should stress that (apparently) there is a weak shoulder in Fig. 4, at about $1.25 T_{g}$, in the diffusion coefficients calculated from the experimental growth rates, in the region where one expects the "decoupling" phenomenon to occur. However, the kink is only apparent in Reinsch's data ${ }^{6}$ (Fig. 2 ) and the inflection is too faint and uncertain to really demonstrate decoupling. For instance, (i) if one tries to fit the screw dislocation equation to the crystal growth rate data for temperatures above the kink, the results are almost coincident with those for all data points shown in Fig. 3 (the fitted $\lambda$ are again 3.20 and $1.04 \AA$ with the Eyring and StokesEinstein equations, respectively); (ii) the kink has the same order of magnitude as the difference between the data of the two authors that measured $u(T)$ in that temperature range, and is not perceptible in the data of Briggs and Carruthers ${ }^{4}$ (Fig. 2); and finally (iii) one expects that for real decoupling — such as those reported in Refs. 1-3-below $T_{d}$ the Stokes-Einstein or Eyring equation would not describe the diffusion coefficients $D_{u}$ but here they do. In other words, decoupling leads to a permanent change in slope and not only a kink in the $\log D$ vs $1 / T$ curve. In summary, we believe that the kink, if really confirmed, may sign to some other phenomenon (e.g., change of crystal morphology, change of growth mechanism, etc.), but the present results are far from proving decoupling and, in any case, this is not the focus of this paper. We will discuss this particular issue in more detail somewhere else. ${ }^{26}$

\section{CONCLUSIONS}

For diopside the screw dislocation model describes reasonably well (but not perfectly) both the magnitude and temperature dependence of the crystal growth rates in a wide range of undercoolings. The effective diffusion coefficients calculated from crystal growth kinetics and viscosity are congruent with direct measurements of $\mathrm{Si}^{4+}$ and $\mathrm{O}^{2-}$ selfdiffusion. These combined diffusivities cover ten orders of magnitude. $\mathrm{Si}^{4+}$ and $\mathrm{O}^{2-}$ move at the same rate, controlling the transport dynamics involved in crystal growth and viscous flow in diopside liquid. Despite a (possible) weak shoulder on the crystal growth rates at $T \sim 1.25 T_{g}$, the Stokes-Einstein and Eyring equations are valid from the equilibrium liquid down to $1.1 T_{g}$. This study not only reveals the transport mechanism in this complex silicate, but also validates the use of viscosity to account for the transport term of the crystal growth expression in a wide temperature range. It is surprising that one can quite accurately estimate the whole crystal growth rate curve of this silicate liquid using independently measured physical parameters, such as the oxygen anion diameter, the viscosity and the thermodynamic driving force. It would be important to perform similar analyses for other liquids and glasses to ascertain whether or not the present findings can be generalized.

\section{ACKNOWLEDGMENTS}

The authors are greatly indebted to Dr. Vladimir M. Fokin of the Vavilov State Optical Institute, St. Petersburg, Russia, for providing them with his recent (as yet unpublished) data on viscosity; to Natalia Borisova, from the Institute of Silicate Chemistry, St. Petersburg, Russia, for the measurement of the thermodynamic driving force; and to Dr. J. W. P. Schmelzer, of Rostock University, Germany, for critically reviewing this manuscript. Financial support of the Brazilian agencies CAPES, CNPq, Pronex, and FAPESP are highly appreciated.

${ }^{1}$ X. P. Tang, U. Geyer, R. Busch, W. L. Johnson, and Y. Wu, Nature (London) 402, 160 (1999).

${ }^{2}$ A. L. Greer, Nature (London) 402, 132 (1999).

${ }^{3}$ J. W. P. Schmelzer, R. Müller, J. Möller, and I. S. Gutzow, J. Non-Cryst. Solids 315, 144 (2003), and references cited therein.

${ }^{4}$ J. Briggs and T. G. Carruthers, Phys. Chem. Glasses 17, 30 (1976).

${ }^{5}$ E. D. Zanotto, J. Non-Cryst. Solids 130, 217 (1991).

${ }^{6}$ S. Reinsch, Ph.D. Thesis, Technical University of Berlin, 2001, in German.

${ }^{7}$ R. J. Kirkpatrick, G. R. Robinson, and J. F. Hays, J. Geophys. Res. 81, 5715 (1976).

${ }^{8}$ V. M. Fokin and N. S. Yuritsin (unpublished).

${ }^{9}$ K. A. Jackson, in Growth and Perfection of Crystals, edited by R. H. Doremus, B. W. Roberts, and D. Turnbull (Wiley, New York, 1958).

${ }^{10}$ L. L. Burgner and M. C. Weinberg, Phys. Chem. Glasses 42, 184 (2001).

${ }^{11}$ L. W. Herron and C. G. Bergeron, Phys. Chem. Glasses 19, 89 (1978).

${ }^{12}$ M. L. F. Nascimento, Ph.D. Thesis, Federal University of São Carlos, Brazil, 2004, in Portuguese.

${ }^{13}$ N. V. Borisova (unpublished).

${ }^{14}$ J. W. P. Schmelzer, O. V. Potapov, V. M. Fokin, R. Müller, and S. Reinsch, J. Non-Cryst. Solids 333, 150 (2004).

${ }^{15}$ T. Licko and V. Danek, Phys. Chem. Glasses 27, 22 (1986).

${ }^{16}$ S. Kozu and K. Kani, Am. Ceram. Soc. Bull. 23, 377 (1944).

${ }^{17}$ R. S. McCaffery, C. H. Lorig, I. N. Goff, J. F. Oesterle, and O. O. Fritsche, AIME, Techn. Publ. 383, 1 (1931).

${ }^{18}$ D. R. Neuville and P. Richet, Riv. Staz. Sper. Vetro 21, 213 (1990).

${ }^{19}$ A. Sipp, Y. Bottinga, and P. Richet, J. Non-Cryst. Solids 288, 166 (2001).

${ }^{20}$ H. Taniguchi, Contrib. Mineral. Petrol. 109, 295 (1992).

${ }^{21}$ V. P. Klyuev (unpublished).

${ }^{22}$ J. E. Reid, B. T. Poe, D. C. Rubie, N. Zotov, and M. Wiedenbeck, Chem. Geol. 174, 77 (2001).

${ }^{23}$ T. Dunn, Geochim. Cosmochim. Acta 46, 2293 (1982).

${ }^{24}$ N. Shimizu and I. Kushiro, Geochim. Cosmochim. Acta 48, 1295 (1984).

${ }^{25}$ N. Shimizu and I. Kushiro, in Physical Chemistry of Magmas, edited by L. L. Perchuk and I. Kushiro (Springer, Berlin, 1991).

${ }^{26}$ S. Reinsch, R. Müller, M. L. F. Nascimento, and E. D. Zanotto, Proceedings of the 20th International Cong. Glass, Kyoto, 2004. 